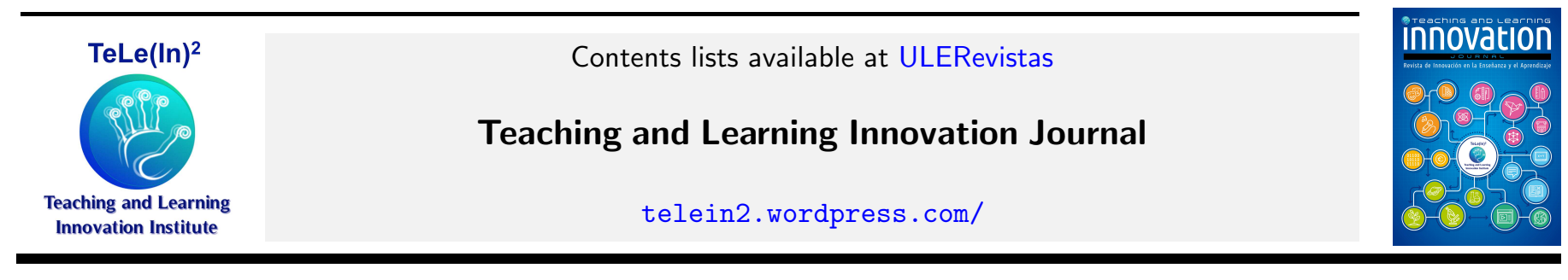

\title{
El uso de la magia como recurso docente: el taller de la magia de la Economía Using magic as a teaching resource: the workshop of the magic of Economics
}

\author{
Juan Carlos Aguado Franco ${ }^{\mathrm{a}}$ \\ ${ }^{a}$ Fundamentos del Análisis Económico, Universidad Rey Juan Carlos, Madrid, Spain
}

\begin{abstract}
En el taller de la magia de la Economía, cuya VIII edición tendrá lugar en noviembre de 2016 en la Universidad Rey Juan Carlos en el marco de la Semana de la Ciencia, se explica la Economía a través de trucos de magia. Se trata de un recurso didáctico ya utilizado anteriormente en matemáticas (matemagia) y que cuenta con una gran aceptación por parte del público pues ayuda a comprender mejor los distintos conceptos explicados y a captar la atención del espectador. El éxito de este taller se plasma en haber alcanzado en algunas ediciones las 700 inscripciones, con todo tipo de público, desde alumnos de bachillerato, alumnos universitarios, y público en general.

Abstract- In the workshop of the magic of Economics, whose eighth edition will take place in November 2016 at the Rey Juan Carlos University in the framework of the Week of Science, Economics will be explained through magic tricks. It is an educational resource previously used in mathematics and has a great acceptance by the public as it helps to better understand the various concepts explained and to capture the viewer's attention. The success of this workshop is reflected in some editions have reached the 700 entries, with all kinds of people, from high school students, university students and the general public.
\end{abstract}

Keywords: magia, innovación docente, Economía magics, Economics, teaching innovation

\section{Introducción}

En el ámbito de las matemáticas se utiliza desde hace ya bastante tiempo el concepto de la matemagia. Consiste la matemagia en aunar dos campos que tienen bastantes similitudes; un mago muestra un hecho sorprendente, inexplicable, mientras que un matemático explica hechos misteriosos, que en muchas ocasiones podrían llegar a parecer mágicos.

Algunas de las ventajas de utilizar la matemagia en clase que se han señalado en distintas publicaciones es que los juegos matemáticos fascinan a mucha gente y por consiguiente permiten atraer la atención de los alumnos; hace que se salga de la rutina habitual y combate el aburrimiento; fomenta un espíritu crítico e imaginativo, pues para encontrar la solución se requiere plantear ideas originales; se fundamentan en aplicaciones muy simples, en general unos cálculos aritméticos, combinatorios y probabilísticos básicos, y unos desarrollos algebraicos al al-

Email address: juancarlos.aguado@urjc.es (Juan Carlos Aguado Franco) cance de cualquier persona sin necesidad de poseer unos conocimientos matemáticos muy elevados.

En los congresos de didáctica de las matemáticas es muy habitual observar la presentación de comunicaciones y ponencias relacionadas con la matemagia.

Esas ventajas pueden ser extrapolables a otros campos de conocimiento, y una experiencia en la que eso se ha llevado a cabo es lo que vamos a exponer en este trabajo. La Economía es uno de esas áreas, pues en el estudio y la docencia de la Economía utilizamos las matemáticas como un instrumento fundamental. Pero la magia puede ser un instrumento útil no solamente en áreas cercanas a las matemáticas; en cualquier campo del conocimiento se puede utilizar la magia como un recurso que consiga captar la atención de los espectadores -léase alumnos en el caso que nos ocupa habitualmente-.

El profesor puede utilizar la magia como un recurso educativo, realizándola él mismo, o puede involucrar a los alumnos con sencillas prácticas que puedan llevar a cabo ellos mismos. En este último sentido, Conde A. (2013) destaca como principales competencias y habili- 
dades físicas y cognitivas que se desarrollan con la magia las siguientes:

- Psicomotricidad fina y gruesa (para manipular y realizar las sutiles y precisas técnicas que esconden nuestros secretos mágicos y moverse acorde a lo que estamos haciendo).

- La memoria (para retener los pasos a seguir, realizar los juegos o memorizar la presentación, el timing y la charla). II Congreso de Innovación Docente Universitaria Telein2

- El pensamiento lógico-matemático (muchos principios que rigen nuestra magia son operaciones y principios matemáticos).

- El pensamiento emocional (el mago debe transmitir diferentes emociones a través de su magia y debe conseguir que los espectadores sientan esas emociones).

- Enfrentarse al fracaso: Es necesario desarrollar en los alumnos la necesidad de enfrentarse a los miedos y no rechazarlos u ocultarlos.

- Creatividad, imaginación, dramatismo y teatralidad (el mago inventa la historia que rodea al desarrollo del juego, es el narrador de historias fascinantes).

- Relaciones interpersonales: al comunicarnos con los espectadores de forma activa.

- Autoestima y seguridad en si mismo, ayudando a superar la timidez.

- Desarrollo del espíritu critico: al enseñarles a desconfiar de las apariencias.

- Capacidad de concentración.

- Desarrollo de la coordinación.

- Desarrollo del concepto de generosidad y altruismo.

- Desarrollo de la expresión oral y mas concretamente de la oratoria en publico.

- Desarrollo del autoconcepto.

\section{Contexto}

En el mes de noviembre de 2016 va a celebrarse la VIII edición del taller de la Magia de la Economía en el marco de la Semana de la Ciencia, en la Universidad Rey Juan Carlos, tanto en el campus de Vicálvaro como en el de Aranjuez, en días diferentes.

Este taller, que nació de forma experimental hace algunos años con unas pocas decenas de alumnos en un único campus de la URJC, ha tenido un crecimiento muy notable, llegando a recibir en las ediciones de 2014 y 2015 a 500 y 700 espectadores respectivamente, si bien podrían haber sido muchos más dado que en ambos casos se tuvieron que limitar las inscripciones por haberse alcanzado rápidamente el aforo máximo de los salones de actos reservados para estas actividades.

Respecto a los lugares en los que se ha llevado a cabo, partiendo de esa experiencia seminal en el campus de Vicálvaro, en el que se ha hecho cada año, se ha representado también en distintas ocasiones tanto en el campus de Fuenlabrada como en el de Móstoles, y en 2015 se presentó por primera vez en el campus de Aranjuez de la Universidad Rey Juan Carlos.

De hecho, ya está programada una edición, financiada por una entidad bancaria española dentro de su programa de formación en aspectos financieros, en la provincia de Guadalajara para el año 2017.

La mayor parte de las ediciones se ha llevado a cabo en el marco de la Semana de la Ciencia o la Noche Europea de los Investigadores.

La actividad que se presenta en este artículo está dirigida a todos los públicos. Así, el público asistente al taller en las diversas ediciones llevadas a cabo es muy variado y está compuesto desde por ancianos que vienen a pasar el rato con curiosidad por el nombre del taller, hasta estudiantes universitarios de muy variadas titulaciones -no solamente del área económica- o estudiantes de instituto -generalmente de primero o segundo de bachillerato- que vienen en una actividad escolar organizada por sus profesores. De hecho, hay institutos que repiten año tras año.

Con este taller se pretende mostrar que se puede aprender la Economía de una forma amena, divertida y diferente, sin por ello perder rigor en las explicaciones.

Como afirma Chaves (2008), para muchas personas el funcionamiento de la economía y el lenguaje que utilizan los expertos en la materia para explicar dicho funcionamiento resultan esotéricos y mágicos. Sin embargo, no es ese aspecto mágico el hecho que justifica este estudio.

Así, en el taller de la magia de la Economía, pretendemos justo lo contrario; intentamos que esos conceptos que a algunos pueden resultar abstractos, enrevesados, incomprensibles y lejanos resulten finalmente accesibles, entendibles y cercanos con la ayuda de la magia.

Efectivamente, la magia constituye un recurso educativo muy poderoso; como señala Ruiz (2013) son muy importantes las posibilidades que nos ofrece ésta herramienta para inducir a la motivación, atención e incluso fascinación de nuestros alumnos, así como para el desarrollo de habilidades cognitivas y comunicativas.

Sin ánimo de exhaustividad, Rodríguez (2013) distingue entre diferentes tipos de recursos educativos inspirados de algún modo en la magia: juegos de lógica, juegos matemágicos y las ilusiones ópticas o engaños visuales.

Las ilusiones ópticas se dividen a su vez en tres tipos: literales, fisiológicas y cognitivas:

- Literales. Creemos ver imágenes que son diferentes a los objetos existentes en la realidad, como se produce por ejemplo con los espejismos, los hologramas, con 
determinadas formas geométricas, etc.

- Fisiológicas. Se producen cuando se altera la percepción debido a la excesiva información visual, como se produce con la repetición de figuras, líneas, matrices o debidas al excesivo brillo, contraste, etc. Ocurren generalmente por errores de la conexión entre ambos hemisferios del cerebro.

- Cognitivas. Depende de factores subjetivos, vemos figuras que no existen, pero asumimos inconscientemente que están presentes.

Precisamente en el taller de la magia de la Economía utilizo una ilusión óptica o engaño visual como metáfora cuando explico que existen ineficientes que se producen en la asignación de los recursos económicos en el momento en el que se aplican medidas redistribuidas de la renta, como impuestos, etc.

En el taller de la magia de la Economía se explican conceptos clave de la Economía. En cada edición del taller los conceptos seleccionados pueden variar, pero los que aparecen con mas frecuencia en todas las ediciones llevadas a cabo son los siguientes:

- el concepto económico de "escasez", que se analiza tanto a nivel de los individuos particulares -consumidore empresas, etc.- como a escala planetaria,

- la creación de dinero bancario y el grave problema que aparece cuando se pierde la fe en el sistema financiero,

- las ineficiencias que surgen cuando se utilizan políticas redistributivas basadas en la fijación de impuestos u otros instrumentos de política económica,

- la necesidad de que exista información perfecta para que se produzca una asignación eficiente de los recursos en la Economía.

Explicaremos a continuación de qué manera se lleva a cabo una explicación de uno de esos conceptos. Lógicamente, no podría ser el objetivo de este trabajo explicarlos todos ellos de forma detallada, pues la extensión de ese trabajo sería excesiva. Relataremos aquí por tanto una explicación que se lleva a cabo en el taller utilizando una ilusión óptica, que es uno de los recursos educativos inspirados de algún modo en la magia, como señalábamos en el epígrafe anterior, con la ayuda de la figura 1, que consta a su vez de otras dos figuras, una rectangular a la izquierda y otra cuadrada a la derecha, formadas ambas por dos trapecios y dos rectángulos.

Hay que señalar que el origen de este truco geométrico está adjudicado a Sam Loyd, uno de los mayores creadores de acertijos y pasatiempos de todos los tiempos (Muñoz, 2004), pero la argumentación económica de la explicación es del autor de este artículo.

El planteamiento, aplicándolo a la enseñanza de la Economí sería el siguiente.
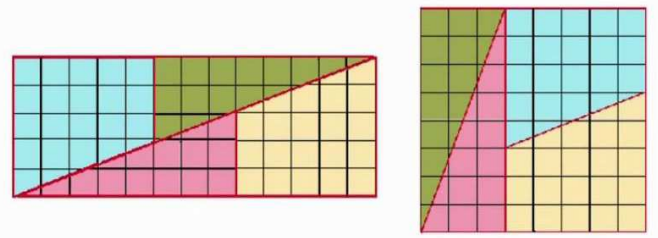

Figure 1: Un rectángulo y un cuadrado.

Suponemos que podemos ordenar a las personas en función de la renta que perciben, y establecer una distinción por tanto entre quienes ganan más y quienes ganan menos dinero. De este modo, la renta que obtienen las personas con un mayor poder adquisitivo queda reflejada en el eje de abscisas de la gráfica de la izquierda, mientras que la renta que perciben las personas que menos renta reciben quedaría contabilizada en el eje de ordenadas de esa misma gráfica (es decir, los más favorecidos reciben 13 y los más desfavorecidos tan solo 5 , que son los cuadraditos que podemos contar en horizontal y en vertical en la figura, respectivamente, dando lugar a una distribución claramente desigual de la renta).

El total de cuadraditos que aparecen en la figura sería por tanto de $5 \times 13=65$.

Obsérvese que ese planteamiento es totalmente similar al que se emplea cuando se representa la tradicional curva de Lorenz, representada en la figura 2, que es una forma de plasmar las desigualdades en la percepción de renta teniendo en cuenta los distintos percentiles de la población, que previamente ha sido ordenada de manera creciente en función de la renta percibida.

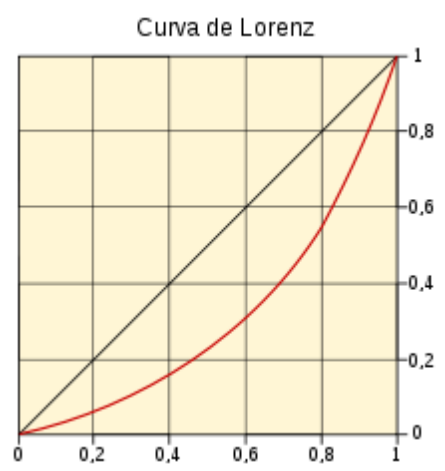

Figure 2: La curva de Lorenz.

Si suponemos a continuación que introducimos medidas redistributivas de la renta, de forma que hacemos pasar renta de los individuos más favorecidos a los más desfavorecidos, alterando la forma de la figura geométrica resultante -el rectángulo del que partimos-, pero utilizando las mismas áreas -triángulos y trapecios- con las que contábamos níanteriormente, vemos en la parte derecha de la gráfica que tanto unos como otros tipos de individuos perciben 8 (se 
trata de un cuadrado en el que tanto en abscisas como en ordenadas podemos contar 8 cuadraditos).

Habríamos avanzado por tanto hacia una distribución de la renta totalmente igualitaria, (que se correspondería en la curva de Lorenz de la figura 2, por continuar con la comparación, con una curva que se solapara totalmente con la recta de equidistribución).

Si nos fijamos en la parte de la derecha dentro de la figura 1 , y contamos los cuadraditos, vemos que tan solo hay $8 \times 8=64$. Es decir, que con las mismas áreas coloreadas que teníamos anteriormente, con las que teníamos 65 cuadraditos, simplemente reordenándolas de otra manera diferente ahora tan solo tenemos 64 .

Hay que remarcar en este punto que para componer el cuadrado de ocho cuadraditos de lado se utilizan exactamente las mismas figuras, -triángulos y trapecios- que se empleaban al formar el rectángulo que tenía lados de 5 y 13 cuadraditos respectivamente, simplemente girándolas y componiendo una forma distinta al agruparlas: un cuadrado.

Al hacerlo, misteriosamente ha desaparecido un cuadradito; "como por arte de magia". Este hecho tiene una explicación basada en la geometría, debido a que la pendiente de las hipotenusas de los triángulos no es la misma en los distintos triángulos, por lo que las áreas quedarían mínimamente solapadas en determinados lugares, algo casi imperceptible a simple vista.

Pero no es cuestión de explicar en este momento geometría, sino de buscar la explicación económica que justificaría esa pérdida, dando por sentado que se ha producido la magia de que 65 cuadraditos se han convertido en 64 ; ha desaparecido misteriosamente uno.

En efecto, cuando fijamos impuestos sabemos que se produce una ineficiencia en la asignación de los recursos (lo que recauda el Estado es menor que lo que pierden conjuntamente los consumidores y los productores, porque los primeros pagan un precio mayor y consumen menos unidades, y los segundos venden menos y raramente consiguen una traslación perfecta del impuesto a los consumidores por lo que acaban asumiéndolo en parte).

Los trucos de magia que se utilizan en el taller no sólo están relacionados con figuras geométricas como el descrito en los párrafos precedentes.

Así, para ilustrar el concepto de "escasez", se hacen saltar a palillos, supuestamente con telequinesia, y se convierte mágicamente un trozo de papel en un billete de 50 euros únicamente con el soplido del mago.

Se muestra igualmente cómo se pueden imprimir con una pequeña prensa billetes de curso legal a partir de trozos de folio de diferente tamaño, y cuál sea su tamaño repercutirá en el valor del billete impreso.

Se crea también dinero -monedas- simplemente con el paso de mano a mano de un billete, para ilustrar el proceso de creación de dinero bancario. Eso sí, el dinero que se crea en esta ocasión es falso, es de chocolate, como tampoco es real el dinero que crean los bancos comerciales a través de los préstamos que realizan.
Se hacen trucos con cuerdas, cuyo tamaño misteriosamente cambia a lo largo de la representación, en función de las necesidades de la explicación que el profesor-mago está desarrollando.

Finalmente, los trucos probablemente más espectaculares se realizan en la parte final del espectáculo cuando se explica la necesidad de que exista información perfecta para que la asignación de los recursos sea eficiente.

Estos trucos finales son de cartas. En ellos, el profesormago adivina la carta seleccionada por voluntarios que salen al escenario de muy diversas formas, e incluso es capaz de introducirse en la mente de alguno de ellos para obligarle a discriminar correctamente si cada una de las cartas de la baraja -se utiliza como es habitual en la magia una baraja francesa- es de color negro o rojo.

Se incorpora en alguno de estos trucos también el azar, a través de un dado, para que la adivinación del profesormago resulte aún más complicada.

Se muestra de esta forma que la información de la que disponen los distintos actores involucrados en el desarrollo del juego es diferente -obviamente debido al hecho de que con sus poderes adivinatorios el mago es capaz de tener una información de la que carecen los demás-. Esto, extrapolándolo a lo que ocurre en Economía, conduce a resultados ineficientes cuando algunos agentes económicos tienen información privilegiada, o resulta demasiado costoso obtener información para la toma de decisiones económicas, es decir cuando hay información imperfecta.

\section{Resultados}

Podemos analizar los resultados desde dos puntos de vista; el primero de ellos consistiría en analizar el éxito de la experiencia en función del número de asistentes al taller; el segundo, desde el logro educativo que supone la experiencia, es decir, si verdaderamente ayuda a que la asimilación de los contenidos por parte de los alumnos y se consiga mantener la atención de los mismos.

Desde el primer punto de vista, es obvio que el crecimiento de los asistentes al taller ha sido espectacular, dado que en la primera experiencia fueron tan solo 40 personas, y congregar a 700 personas en una actividad de este tipo, colgando el cartel de aforo completo es ciertamente un hecho destacable. Es necesario señalar que hay algunos factores que pueden incidir en este dato: la oferta de créditos ECTS para los alumnos y la propaganda que se lleve a cabo.

A este respecto hay que señalar que al tratarse de una actividad de muy corta duración -no llega a las dos horas-, no ofrece más que 0,1 créditos ECTS, por lo que ese estímulo parece poco relevante. De hecho, para VIII edición del taller ya no se ofrecerá el reconocimiento académico de créditos.

Respecto a la propaganda efectuada, la fijación de carteles en la Universidad se ha ido reduciendo paulatinamente y en el año 2015 se eliminó por completo. No obstante, 
a través del blog del profesor y de su cuenta de twitter se informa de la celebración del evento, del mismo modo que se puede obtener información a través del portal de la Comunidad de Madrid encargado de la difusión de la Semana de la Ciencia.

La otra parte de la evaluación de los resultados no ha sido abordada. En efecto, resulta complicado poder cuantificar en qué medida la asistencia al taller ha ayudado a mejorar la asimilación de los contenidos a las personas asistentes al mismo, salvo que se les hiciera un examen de conocimientos previos antes de entrar y otro de conocimientos finales a la salida, algo que parece inviable.

Finalmente, hay que señalar que las técnicas descritas son utilizadas no solamente en el taller, sino que también son incorporadas a la docencia habitual en las aulas. Medir su impacto también sería muy complicado, por lo que tenemos que quedarnos únicamente con la satisfacción expresada informalmente por parte de los alumnos, que es ciertamente gratificante.

\section{Conclusiones}

Aunque el origen esté en la matemagia, la extrapolación de estas prácticas, consistentes en utilizar la magia en las explicaciones, a otras áreas del conocimiento, cercanas o no a las matemáticas, es posible e incluso nos atreveríamos a decir recomendables.

Es fundamental, en cualquier caso, dar respuesta a las preguntas acerca de por qué, el cómo, el para qué y el cuándo utilizar la magia en la labor docente (Ruiz, 2013).

En efecto, se trata de utilizar un recurso más, ciertamente atractivo, pero siempre con una finalidad clara de apoyo en el proceso de enseñanza-aprendizaje.

Finalmente, parafraseando a Rodríguez (2013), los profesores y profesoras que hacen MAGIA son: Motivadores, Amenos, Generosos, Imaginativos y Audaces.

\section{Referencias}

[1] Almau, A. (2013). Se puede enseñar matemáticas o física con trucos de magia. Cuadernos de Pedagogía n ${ }^{\circ}$ 433. 38-43. Recuperado de: https://convivencia.files.wordpress.com/2013/09/cuadernos433.pdf

[2] Chaves, J.A. (2008) La magia de la Economía. La Nación 26/10/2008. Recuperado de: http://www nacion.com/opinion/foros/magiaeconomia_0_1009299128.html

[3] Conde, A. (2013). La magia como herramienta pedagógica. Recuperado de: http://alvarocondemago.blogspot.com.es/2013/09/la-magia-como-herramienta-pedagogica.html

[4] Muñoz, J. (2004). Una matemática motivadora: la matemagia. VI Jornadas de Educación matemática de la Comunidad Valenciana. Recuperado de: http://thales.cica.es/ estalmat/Actividades-ejemplos/MatemagiaEstalmat.pdf

[5] Ruiz, X. (2013). Educando con magia. El ilusionismo como recurso didáctico. Madrid: Narcea.

[6] Rodríguez, S. (2013): La magia de enseñar: el docente como ilusionista. Recuperado de: http://www. salvarojeducacion.com/2013/02/la-magia-de-ensenar-el-docente-como.html 